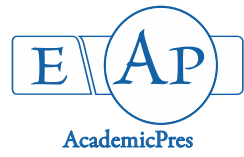

\title{
Regulatory Network Identification, Promoter and Expression Analysis of Arabidopsis thaliana NPR1 in Defense Responses against Stresses
}

\author{
Amir G. SHAHRIARI ${ }^{1 *}$, Aminallah TAHMASEBI ${ }^{2}$, Sima SAZEGARI ${ }^{3}$ \\ ${ }^{1}$ Higher Education Center of Eghlid, Department of Agriculture and Natural Resources, Hafez Street, Eghlid, \\ Iran;Shabriari.ag@eghlid.ac.ir ( ${ }^{*}$ correspondingauthor) \\ ${ }^{2}$ Shiraz University, Plant Virology Research Center, Shiraz, Bajgah Iran; Tahmasebi.info@yahoo.com \\ ${ }^{3}$ Ferdowsi University of Mashhad, Department of Plant Breeding and Biotechnology, Iran; ssazegari@yahoo.com
}

\begin{abstract}
Salicylic acid (SA) and jasmonic acid (JA) phytohormones have been known for their roles in plant defense behaviour against biotic and abiotic stresses. They regulate defense pathways by antagonistic interaction. NPR1 as a key regulatory factor in the cross-talk between SA and JA, signaling is essential for the inhibition of JA-responsive gene expression by SA. In silico promoter analysis of $1.5 \mathrm{~kb}$ promoter regions of NPR1 gene revealed that NPR1 contains 23 MYB and 20 WRKY transcription factor binding sites. Different cis-elements associated with various stress responses were identified in Arabidopsis thaliana NPR1. The most common element was allocated to the defense responses against biotic stresses. Based on gene network analysis, NPR1, TGA2 and TGA3 were predicted to have functional cooperation with each other. Affymetrix microarray data analysis of $A$. thaliana under SA treatment demonstrated that most genes involved in NPR1 network are upregulated under SA treatment. Therefore, interaction and cooperation between these factors might serve to fine-tune regulation of defense and immune responses against biotic and abiotic stresses.
\end{abstract}

Keywords: abiotic and biotic stresses; Arabidopsis thaliana; gene network; in silico analysis; NPR1; regulatory elements

\section{Introduction}

Plants are exposed to various biotic and abiotic stresses. Perception of stress signals often results in the biosynthesis of signaling molecules like jasmonic acid (JA) and salicylic acid (SA) phytohormones (Turner et al., 2002; Shah, 2003). Production of these hormones mediates signal transduction cascades that lead to physiological adaptation of the plant to stresses (Kunkel and Brooks, 2002).

SA plays a significant role in plant resistance to biotic and abiotic stress factors (Loake and Grant, 2007; Vlot et al., 2009; Miura and Tada, 2014). JA pathway is also involved in different processes including responses to biotic and abiotic stresses (Howe and Jander, 2008; Browse, 2009).

SA and JA exhibit antagonistic interactions in response to plant pathogens. The activation of SA biosynthesis or signaling in many plant-pathogen interactions has been shown to result in the inhibition of JA biosynthesis or signaling through non-expresser of pathogenesis-related genes 1) (NPR) and WRKY70 proteins (Koornneef and Pieterse, 2008; Yasuda et al., 2008; Bari and Jones, 2009; Antico et al., 2012; Thaler et al., 2012). Conversely, induction of the JA pathway represses the SA signaling through MAPK4 and JIN2 factors (Kachroo and Kachroo, 2007; Koornneef and Pieterse, 2008).

Transcription factors (TFs) bind to cis-acting regulatory elements (CAREs) with short conserved nucleotide motifs at the $5^{\prime}$ end of the genes and upstream of the gene transcription start site known as the promoter (de Boer et al., 1999). Plant promoter identification may increase fundamental information in the understanding of the regulation of gene expression (Shahmuradov et al., 2003).

TFs regulate gene expression via interaction with CAREs. The interaction between TFs and cis-acting DNA elements might activate or repress genes (Moore et al., 2011). TFs can interact with jasmonate signaling pathway and mediate response to biotic and abiotic stresses (Century et al., 2008). The role of TFs in hormone signaling can also activate defence genes (Lu et al., 2011).

The NPR1, as a positive regulator of SA, is essential for the inhibition of JA-responsive gene expression by SA (Zhang et al., 1999; Després et al., 2000; Zhou et al., 2000). In the SA pathway, the activity of NPR1 is regulated by several SA-dependent modifications (reviewed in $\mathrm{Fu}$ and Dong, 2013). By interacting with TGACGTCA cis- 
334

element-binding protein (TGA) transcription factors, NPR1 acts as a co-activator of SA-induced gene transcription and activates SA marker genes such as pathogenesis related protein (PR1). It has been shown that expression of NPR1 is regulated by WRKY TFs (Yu et al., 2001). WRKY TFs fine-tune and amplify downstream transcriptional responses (Wang et al., 2006; Eulgem and Somssich, 2007). WRKY factors act as positive regulators of the SA-mediated defenses while these factors repress the JA response functioning (Li et al., 2004). Moreover, several WRKY and TGA TFs have been shown to be important for SA/JA crosstalk and contribute to the complexity of the SA- and NPR1-dependent signaling network (Wang et al., 2006; Pieterse et al., 2012; Gimenez-Ibanez and Solano, 2013). V-myb myeloblastosis viral oncogene homolog (MYB) family has been found to be involved in a variety of biological functions such as biotic and abiotic stresses (Lippold et al., 2009; Segarra et al., 2009), hormone responses (Urao et al., 1993) and plant defense responses (Liu et al., 2008).

The identification of CAREs and their interaction with TFs could give some insight into the plant defense against stresses and presents a comprehensive understanding of the regulation of NPR1 gene expression.

Therefore, in the present study, in silico promoter analysis and affymetrix microarray data analysis of $A$. thaliana under salicylic acid treatment was followed to study NPR1 CAREs, TFs and expression analysis of genes involved in Arabidopsis thaliana NPR1 signaling network which are involved in defense responses against biotic and abiotic stresses.

Due to the pivotal role of transcription factors, such studies will advance the knowledge of host-stress interactions and can further provide the development of new strategies for the plant genetic engineering for management of biotic and abiotic stresses.

\section{Materials and Methods}

\section{Promoter analysis of NPRI gene}

The genomic sequence of NPRI gene from $A$. thaliana (AT1G64280) was applied as a platform in order to recognize the promoter region of the $N P R 1$ gene by using the Phytozome database (http://www.phytozome.net/). After identifying the Arabidopsis NPRI on the chromosome using the BLAST-n algorithm, the 1,500 bp upstream of the start codon (ATG) was taken as a promoter. The upstream region sequences of the NPRI in Arabidopsis was analyzed via the PLANT CARE (http: // bioinformatics. psb.ugent. be /webtools/ plantcar e/html/) and Plant Pan (http:/ / plantpan2 .itps.ncku.edu .tw / promoter. php) databases to predict their key CAREs and the precise location of these elements.

\section{NPRI regulatory network analysis}

In order to study the regulatory network of NPR1, GeneMANIA (http://www.genemania.org) database was used to identify different categories of co-expressed genes, physically interacted genes and functionally related genes with NPR1 regulatory factor. Also, STRING 9.0 (http://string-db.org) was used to predict all the proteins that interact with the NPR1 protein in $A$. thaliana.

\section{NPR1 chromosome map}

Chromosome map of $A$. thaliana NPRI was constructed by Chromosome Map Tools available at TAIR (https://www.arabidopsis.org/jsp/ChromosomeMap/tool.j $\mathrm{sp)}$. data

Expression analysiss of NPRI gene based on microarray

To analyze the expression pattern of NPRI and coregulated genes, affymetrix microarray data (http://bar.utoronto.ca database) was used. The relative expression ratio of genes under $10 \mathrm{mM}$ SA treatment was obtained after 3 hours post treatment with SA based on $\log 2$ (treated/control) ratio of three biological replicates.

\section{Results and Discussion}

Figs. 1 and 2 show the exon-intron arrangement and promoter map of NPRI in A. thaliana. NPRI contains 4 exons with transcript length of 2,522 bps, translation length (peptide sequence) of 593 residues and genomic sequence of 2,819 bps. In silico chromosome mapping of NPRI gene of A. thaliana is presented in Fig. 3. NPRI gene of $A$. thaliana was shown to be distributed on only 1 out of 5 chromosomes. In $A$. thaliana, chromosome 1 (23,852,74823,855,566 position) harbored NPRI gene.

Different cis-elements associated with various stress responses such as cell death, response to heat, response to bacterium, response to insect, response to wounding, systemic acquired resistance (SAR), defense response to fungi and negative regulation of defense response were shown in $A$. thaliana NPRI (Fig. 4). These results were in accordance with NPR1 regulatory network, which showed that different functional groups predicted based on NPR1 co-regulated gene by Genemania database, play a role in defense response to bacterium, response to salicylic acid, regulation of responses to stress, response to fungus and immune responses (Table 1 ).

The frequency of various regulatory elements with stress-(abiotic or biotic)-responsive function on NPRI promoter suggests that this gene recruits several TFs to mediate gene expression which leads to enhanced resistance to biotic and abiotic stresses.

In another study, a relationship between architecture of promoter sequence and tobacco PR-1a expression has been reported (Lodhi et al., 2008). Therefore, gene expression regulation studies can increase the current knowledge to understand the molecular mechanisms of plant defense response.

On the other hand, it has been shown that TFs play a key role in the response to different stresses with their roles in the regulation of genes expression (Meshi and Iwabuchi, 1995).

According to Fig. 4, the most common element was allocated to SAR function with $52.17 \%$. SAR as a systemic defense response is developed to enhance disease resistance 
against viral, bacterial and fungal pathogens in plants through up-regulation of pathogenesis-related $(\mathrm{PR})$ genes expression (Metraux et al., 1990).

In silico promoter analysis of $1.5 \mathrm{~kb}$ promoter regions of NPRI gene revealed that this gene is significantly enriched in the WRKY and MYB transcription factor binding sites (TFBSs) (Fig. 5, Table 2). Promoter analysis showed that the NPR1 contains $23 \mathrm{MYB}$ and 20 WRKY TFBS (Fig. 5).

WRKY elements with common TGAC signal sequence were observed in NPRI promoter (Table 2).

Analysis of motifs in the promoter of NPRI gene suggests that the TGAC signal sequence may be a target for crosstalk regulation. TGA and WRKY TFs further contribute to the complexity of the SA- and NPR1dependent signaling network involved in SA-dependent SAR (Wang et al., 2006) and some of the WRKY TFs have been implicated as a positive regulator of the SA-mediated defenses ( $\mathrm{Li}$ et al., 2004). Therefore, WRKYs seem to play important roles in the suppression of JA responses. Analysis of protein-protein interaction network is presented in Fig. 6. Based on STRING web server's results, NPR1 mostly interacts with the different types of TGA TFs (Fig. 6).

In addition to promoter analysis of NPRI gene and STRING results, the NPR1 regulatory network was analyzed to identify other genes that participate in this regulatory network. Construction of gene networks can provide a powerful tool for identification of genes involved in biotic and abiotic stress responses (van Verk et al., 2011).

According to the results, NPR1, 2, 3 and 4, TGA2 and 3, NIMIN-2 and 3, and CUL3A, showed physical interaction with each other while only PR1, 2, 3 and 4, TGA3 and CUL3A are expressed together (Fig. 7 and 8). Also, NPR genes including NPR1, 2, 3, 4, 5 and 6 showed common protein domains (Fig. 9). Analysis of NPR protein structure in NCBI-Conserved domain database (https://www.ncbi.nlm.nih.gov/Structure/cdd/wrpsb.cgi) revealed that NPR1, 2, 3 and 4 have three common domains including NPR1, Ankrin and BTB. NPR1 acts as a defense-related domain in Arabidopsis. Ankrin domain mediates the protein-protein interaction which is necessary for NPR protein family by their regulatory role through linking with other proteins (Alvo et al., 2010). Based on the hereby findings, NPR1, TGA2 and TGA3 are suggested to cooperate with each other in a common network (Fig. 10).

NPR1 interacts with TGA TFs, while they can target WRKY TFs. Moreover, WRKY can regulate the expression of NPR1 (Yu et al., 2001). NPR1-dependent crosstalk and TGA TFs can play a role in SA/JA crosstalk. Therefore, it can be hypothesized that NPR1-TFs interactions might play a role in the SA-mediated suppression of JA-responsive gene expression. Similarly, other studies showed that plant hormones biosynthesis and signaling pathways are controlled by different TFs (Jensen et al., 2013; Sakamoto $e t$ al., 2013).

MYB and WRKY families can likely be critical players in responses to biotic and abiotic stresses. The antagonistic effects of SA on the JA signaling pathways are partly dependent on the opposite effects of the transcription factors (Zander et al., 2010).

To study the expression pattern of NPRI and other related genes, expression analysis of co-expressed network genes was assesed based on Arabidopsis microarray data under salicylic acid treatment. These data were extracted from bar.utoronto.ca database. All genes involved in NPR1 regulatory network were up-regulated under SA treatment except for $\mathrm{Cul} 3 \mathrm{~A}$. Cul3A encodes ligase enzymes which participate in degradation of regulatory proteins such as NPR1 (Thomman et al., 2005).

According to the results, the expression ratio of CulA3 is down-regulated under salicylic acid treatment (Table 3). The highest up-regulation ratio belongs to NPR3 (Table 3). The NPR1 and TGA3 relative expression ratios showed the same value. The current findings are in agreement with the results of another study (Yu et al., 2001). Therefore, it seems that NPR1, 3, 4 and TGA3 genes are probably co-regulated. Thus, interaction and cooperation between these factors might fine-tune regulation of defense and immune responses through SA signaling pathway. In another study, $P R$ proteins were up-regulated after wounding and drought stress; this can be due to the presence of stress-related elements in their promoters (Kaur et al., 2017).

Thus, the key role of promoters in controlling gene expression has been confirmed (Marino-Ramirez et al., 2009).

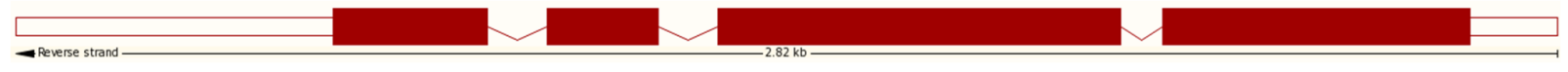

Fig. 1. Exon-intron arrangement of NPRI gene using the Phytozome database. Exons are represented by red boxes and connecting lines represent introns in $A$. thaliana

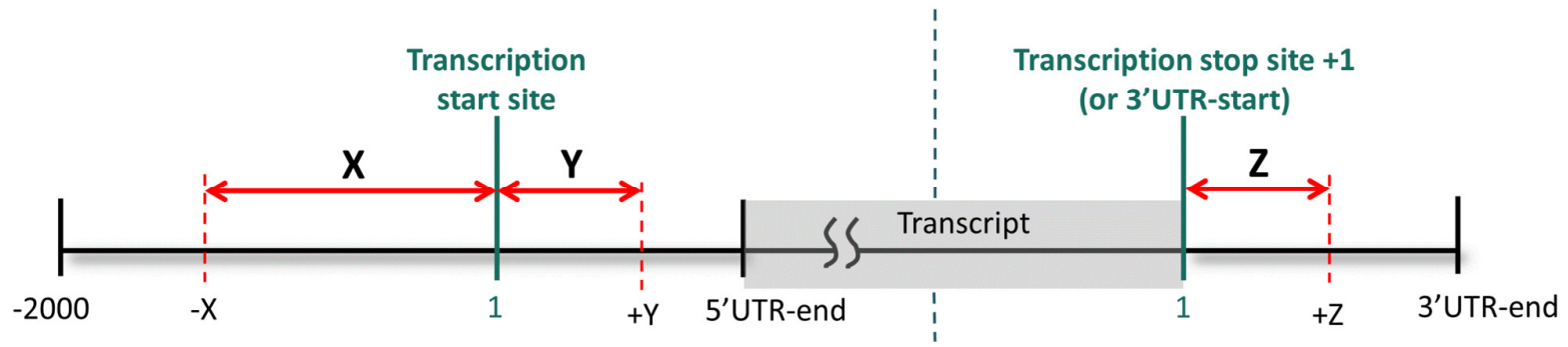

Fig. 2. Upstream and downstream coordinates of $A$. thaliana NPRI promoter obtained using Plant PAN 


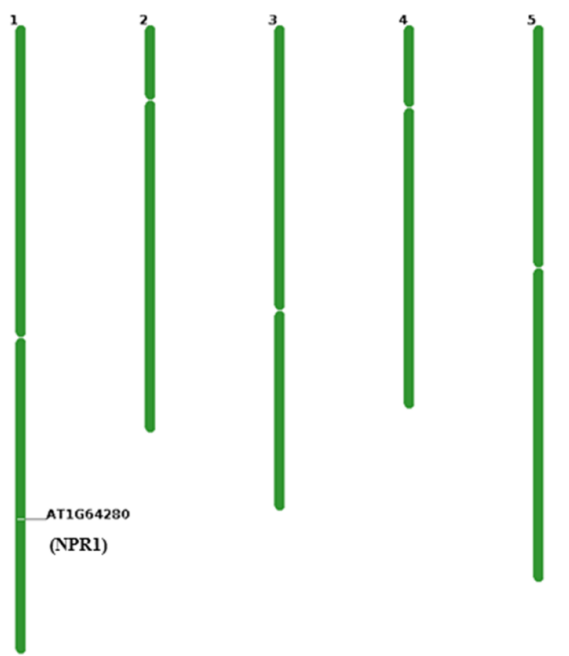

Fig. 3. Chromosomal distribution of NPRI gene in $A$. thaliana, constructed by Chromosome Map Tools available at TAIR

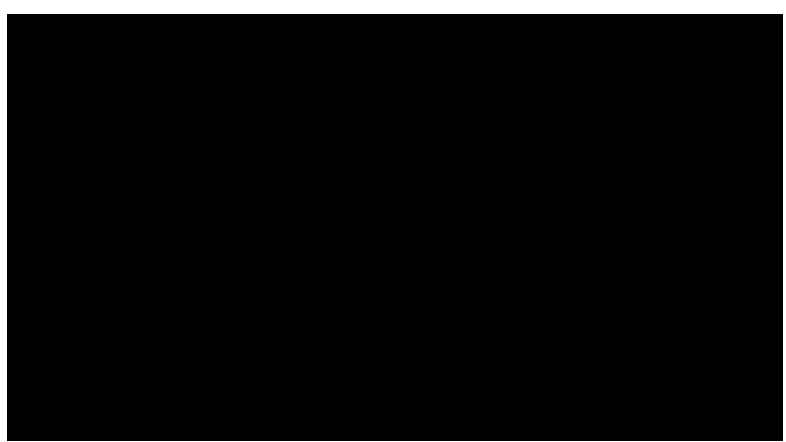

Fig. 5. The TFBS frequencies of MYB and WRKY in NPRI promoter of $A$. thaliana

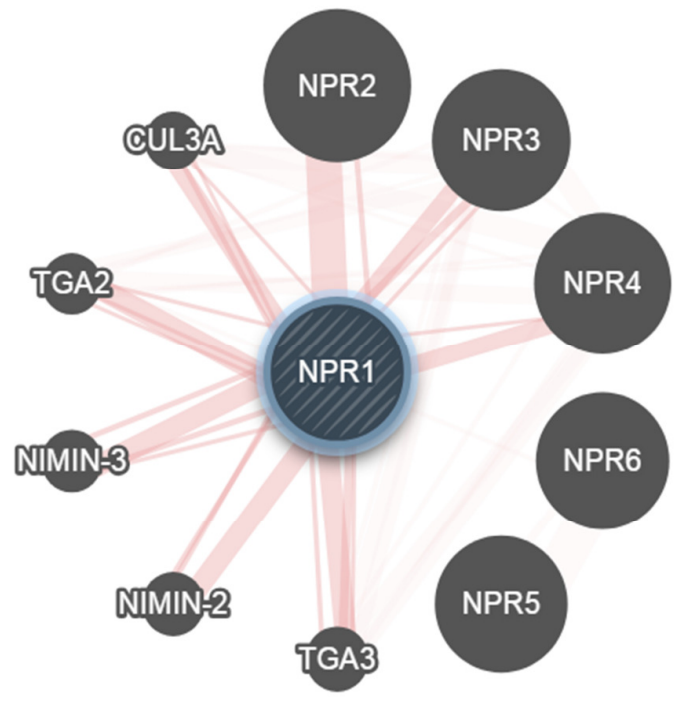

Fig. 7. Physically interacted proteins with NPRI identified using Genemania database

According to the database predictions, gene products are considered as physical interacted if they were found to interact in a protein-protein interaction according to any previous study or report specifically based on proteome-wide binary protein-protein interaction map

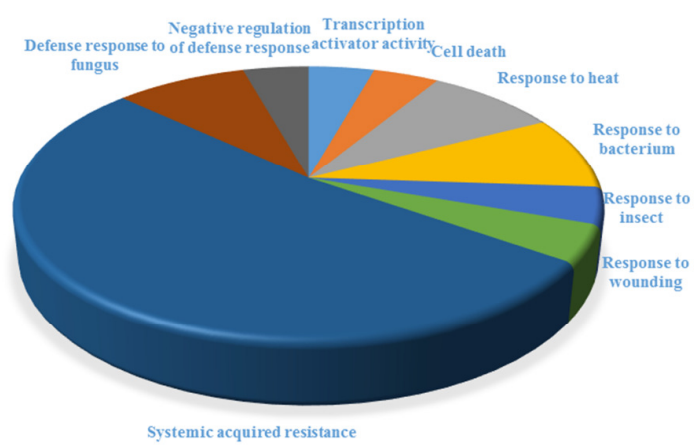

Fig. 4. Pie distribution of identified motifs of $A$. thaliana NPR1 obtained using AtPAN, based on their biological functions

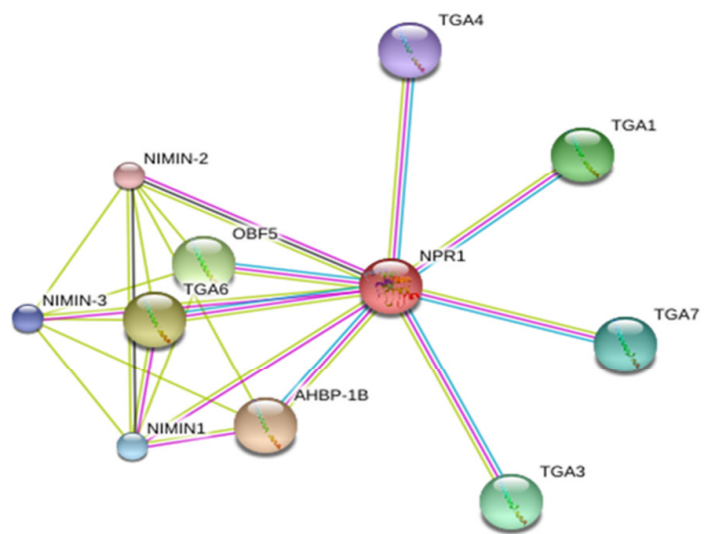

Fig. 6. Protein-protein interaction network analysis of $A$. thaliana NPR1 obtained using STRING database

Protein nodes in the network are automatically highlighted in color. Colored lines between the proteins indicate the various types of interaction evidence. an edge may be drawn with up to 7 differently colored lines - these lines represent the existence of the seven types of evidence used in predicting the associations. Red line - indicates the presence of fusion evidence, Green line - neighborhood evidence, Blue line - cooccurrence evidence, Purple line - experimental evidence, Yellow line - text mining evidence, Light blue line - database evidence, Black line - co-expression evidence

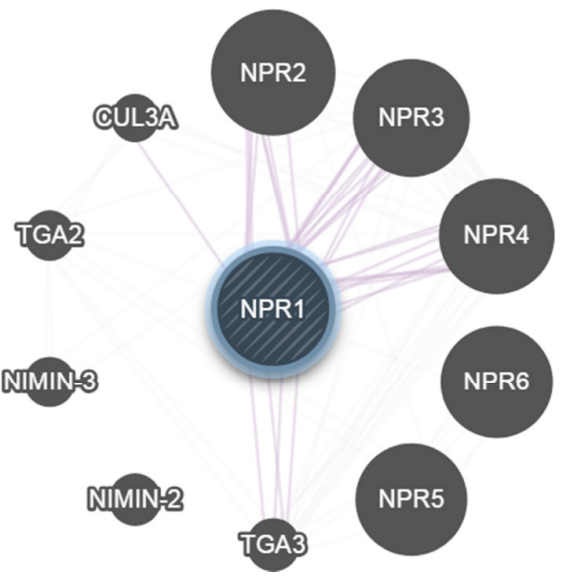

Fig. 8. Regulatory network of NPRI co-expressed genes using Genemania database

The co-expressed genes are linked together if their expression levels are similar across conditions in any reported gene expression study. The data are collected from the Gene Expression Omnibus (GEO) 


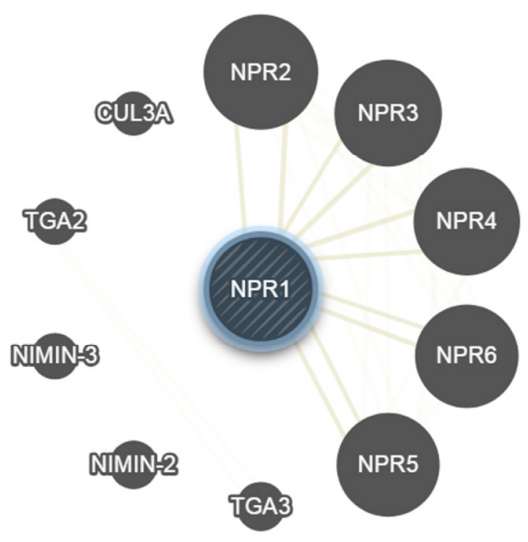

Fig. 9. Shared protein domains in NPRI related genes depicted using GeneMania based on PFAM and INTERPRO domain databases

According to the Genemania analysis, two gene products are linked as shared protein domains if they showed same protein domains

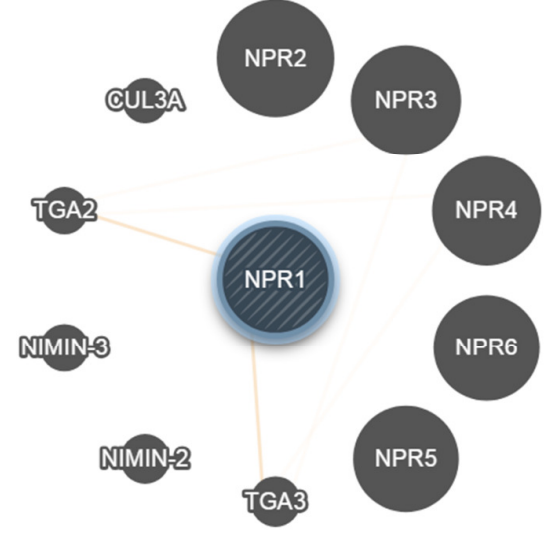

Fig. 10. The genes predicted to have functional relationships with NPRl gene using Genemania

Two proteins are predicted to interact if their orthologs are known to interact in another organism

Table 1. Different functional groups in NPRI regulatory network depicted using Genemania database. The identified genes in NPR1 gene regulatory network were categorized as different functional groups based on their functions in Arabidopsis

\begin{tabular}{cc}
\hline Functional group & Genes \\
\hline Defense response to bacterium & TGA3,NPR3,NPR1,NPR4,TGA1, \\
Response to salicylic acid & TGA3,NPR1,NPR4, \\
Regulation of responses to stresses (biotic and abiotic) & TRX5,NPR1,NPR3,NPR4 \\
Response to fungi & NPR1,NPR3,NPR4 \\
Immune responses & TGA3,NPR1,NPR3, NPR4 \\
\hline
\end{tabular}

Table 2. TFBS of MYB and WRKY in NPR1 promoter of $A$. thaliana

\begin{tabular}{ccc}
\hline Factor & Location & Signal sequence \\
\hline MYBST1 & $295(-)$ & GGATA \\
MYBPLANT & $1,152(-)$ & MACCWAMC \\
MYBCORE & $1,266(+)$ & CNGTTR \\
MYB1AT & $1,431(+)$ & WAACCA \\
MYB2CONSENSUSAT & $1,441(-)$ & YAACKG \\
MYBCORE & $1,441(+)$ & CNGTTR \\
MYBCORE & $1,509(+)$ & CNGTTR \\
WRKY71OS & $766(+)$ & TGAC \\
WRKY71OS & $1,293(+)$ & TGAC \\
WRKY71OS & $1,387(+)$ & TGAC \\
WRKY71OS & $1,392(+)$ & TGAC \\
WRKY71OS & $1,408(-)$ & TGAC \\
\hline
\end{tabular}

Table 3. Expression analysis of NPRI co-expressed genes under salicylic acid treatment. The relative expression ratios are shown as $\log _{2}$ (treated/control) of three biological replicates

\begin{tabular}{cc}
\hline Gene & Relative expression ratio \\
\hline NPR1 & 1.6 \\
NPR2 & 0.11 \\
NPR3 & 3.2 \\
NPR4 & 2.1 \\
TGA3 & 1.6 \\
Cul3A & -0.7 \\
WRKY1 & 0.14 \\
\hline
\end{tabular}


338

\section{Conclusions}

Taken together, identification of interacting proteins constitutes an important step in understanding the mechanism of their activation in defense signaling pathways and their roles in regulating the expression of related genes. Therefore, such analysis can provide new tools for the plant genetic engineering to protect crops against biotic and abiotic stresses.

\section{Acknowledgements}

The present work was supported by the Higher Education Center of Eghlid.

\section{References}

Alvo M, Liu Z, Williams A, Yauk C (2010). Testing for mean and correlation changes in microarray experiments: an application for pathway analysis. BMC Bioinformatics 11:60.

Antico CJ, Colon C, Banks T, Ramonell KM (2012). Insights into the role of jasmonic acid-mediated defenses against necrotrophic and biotrophic fungal pathogens. Frontiers in Biology 7:48-56.

Bari R, Jones JD (2009). Role of plant hormones in plant defence responses. Plant Molecular Biology 69(4):473-488.

Browse J (2009). Jasmonate passes muster: A receptor and targets for the defense hormone. Annual Review of Plant Biology 60:183-205.

Century K, Reuber TL, Ratcliffe OJ (2008). Regulating the regulators: the future prospects for transcription-factor-based agricultural biotechnology products. Plant Physiology 147(1):20-29.

de Boer GJ, Testerink C, Pielage G, Nijkamp HJ, Stuitje AR (1999). Sequences surrounding the transcription initiation site of the Arabidopsis enoyl-acyl carrier protein reductase gene control seed expression in transgenic tobacco. Plant Molecular Biology 39:1197-1207.

Després C, DeLong C, Glaze S, Liu E, Fobert PR (2000). The Arabidopsis NPR1/NIM1 protein enhances the DNA binding activity of a subgroup of the TGA family of bZIP transcription factors. Plant Cell 12:279-290.

Eulgem T, Somssich IE (2007). Networks of WRKY transcription factors in defense signaling. Current Opinion in Plant Biology 10(4):366-371.

Fu ZQ, DongX (2013). Systemic acquired resistance: turninglocal infection intoglobal defense. Annual Review of Plant Biology 64:839-863.

Gimenez-Ibanez S, Solano R (2013). Nuclear jasmonate and salicylate signaling and crosstalk in defense against pathogens. Frontiers in Plant Science 4:72.

Howe GA, Jander G (2008). Plant immunity to insect herbivores. Annual Review of Plant Biology 59:41-66.

Jensen MK, Lindemose S, Masi FD, Reimer JJ, Nielsen M, Perera V, Workman CT, Turck F, Grant MR, Mundy J, Petersen M, Skriver K (2013). ATAF1 transcription factor directly regulates abscisic acid biosynthetic gene NCED3 in Arabidopsis thaliana. FEBS Open Bio 3:321-327.

Kachroo A, Kachroo P (2007). Salicylic acid-, jasmonic acid- and ethylenemediated regulation of plant defense signaling Genetic Engineering
28:55-83.

Kaur A, Kumar Pati P, Maitra Pati A, Kaur Nagpal A (2017). In-silico analysis of cis-acting regulatory elements of pathogenesis-related proteins of Arabidopsisthaliana and Oryza sativa.Plos One 12(9):e0184523.

Koornneef A, Pieterse CMJ (2008). Crosstalk in defense signaling. Plant Physiology 146:839-844.

Kunkel BN, Brooks DM (2002). Cross talk between signaling pathways in pathogen defense. Current Opinion in Plant Biology 5:325-331.

LiJ, Brader G, Palva ET (2004). The WRKY70 transcription factor: a node of convergence for jasmonate-mediated and salicylate-mediated signals in plant defense. Plant Cell 16:319-331.

Lippold F, Sanchez DH, Musialak M, Schlereth A, Scheible WR, Hincha DK, Udvardi MK (2009). AtMyb41 regulates transcriptional and metabolic responses to osmotic stress in Arabidopsis. Plant Physiology 149(4):1761-1772.

Liu L, Du H, Tang XF, Wu YM, Huang YB, Tang YX(2008). The roles of MYB transcription factors on plant defense responses and its molecular mechanism. YiChuan 30(10):1265-1271.

Loake G, Grant M (2007). Salicylic acid in plant defense-the players and protagonists. Current Opinion in Plant Biology 10(5):466-472.

Lodhi N, Ranjan A, Singh M, Srivastava R, Singh SP, Chaturvedi CP, Ansari SA, Sawant SV, Tuli R (2008). Interactions between upstream and core promoter sequences determine gene expression and nucleosome positioning in tobacco PR-la promoter. Biochimica et Biophysica Acta 1779:634644.

Lu J, Ju H, Zhou G, Zhu C, Erb M, Wang X, Wang P, Lou Y (2011). An EAR-motif-containing ERF transcription factor affects herbivore-induced signaling, defense and resistance in rice. The Plant Journal 68(4):583-596.

Marino-Ramirez L, Tharakaraman K, Bodenreider O, Spouge J, Landsman $\mathrm{D}$ (2009). Identification of cis-regulatory elements in gene co-expression networks using A-GLAM. Methods in Molecular Biology 541:1-22.

Meshi T, Iwabuchi M (1995). Plant transcription factors. Plant and Cell Physiology 36:1405-1420.

Metraux JP, Signer H, Ryals J, Ward E, Wyssbenz M, Gaudin J, Raschdorf K, Schmid E, Blum W, Inverardi B (1990). Increase in salicylic-acid at the onset of systemic acquired resistance in cucumber. Science 250(4983):1004-1006.

Miura K, Tada Y (2014). Regulation of water, salinity, and cold stress responses by salicylic acid. Frontiers in Plant Science 5:4.

Moore JW, Loake GJ, Spoel SH (2011). Transcription dynamics in plant immunity. Plant Cell 23(8):2809-2820.

Pieterse CM, Van der Does D, Zamioudis C, Leon-Reyes A, Van Wees SC (2012). Hormonal modulation of plant immunity. Annual Review of Cell and Developmental Biology 28:489-521.

Sakamoto T, Morinaka Y, Inukai Y, Kitano H, Fujioka S (2013). Auxin signal transcription factor regulates expression of the brassinosteroid receptor gene in rice. The Plant Journal 73:676-688.

Segarra G, Van der Ent S, Trillas I, Pieterse CMJ (2009). MYB72, a node of convergence in induced systemic resistance triggered by a fungal and a bacterial beneficial microbe. Plant Biology 11(1):90-96.

Shah J (2003). The salicylic acid loop in plant defense. Current Opinion in Plant Biology 6:365-371. 
Shahmuradov IA, Gammerman AJ, Hancock JM, Bramley PM, Solovyev VV (2003). PlantProm: A database of plant promoter sequences. Nucleic Acids Research 31:114117.

Thaler JS, Humphery PT, Whiteman NK (2012). Evolution of jasmonate and salicylate signal crosstalk. Trends in Plant Science 17:260-270.

Thomann A, Brukhin V, Dieterle M, Gheyeselinck J, Vantard M, Grossniklaus U, Genschik P (2005). Arabidopsis CUL3A and CUL3B genes are essential for normal embryogenesis. The Plant Journal 43(3):437-448.

TurnerV,Ellis JG, Devoto CA (2002). The jasmonate signal pathway. Plant Cell 14(Suppl):S153-S164.

Urao T, Yamaguchi-Shinozaki K, Urao S, Shinozaki K (1993). An Arabidopsis myb homolog is induced by dehydration stress and its gene product binds to the conserved MYB recognition sequence. Plant Cell 5(11):1529-1539.

van Verk MC, Bol JF, Linthorst HJ (2011). Prospecting for genes involved in transcriptional regulation of plant defenses, a bioinformatics approach. BMC Plant Biology 11(1):88.

Vlot AC, Dempsey DMA, Klessig DF (2009). Salicylic acid, a multifaceted hormone to combat disease. Annual Review of Phytopathology 47:177206.

Wang D, Amornsiripanitch N, Dong X (2006). A genomic approach to identify regulatory nodes in the transcriptional network of systemic acquired resistance in plants. PLOS Pathogens 2:1042-1050.

Yasuda M, Ishikawa A, Jikumaru Y, Seki M, Umezawa T, Asami T, Maruyama-Nakashita A, Kudo T, Shinozaki K, Yoshida S, Nakashita $H$ (2008). Antagonistic interaction between systemic acquired resistance and the abscisic acid-mediated abiotic stress response in Arabidopsis. Plant Cell 20(6):1678-1692.
Yu D, Chen C, Chen Z (2001). Evidence for an important role of WRKY DNA binding proteins in the regulation of NPR1 gene expression. Plant Cell 13:1527-1540.

Zander M, La Camera S, Lamotte O, Métraux JP, Gatz C (2010). Arabidopsis thaliana class-II TGA transcription factors are essential activators of jasmonic acid/ethylene-induced defense responses. The Plant Journal 61(2):200-210.

Zhang Y, Fan W, Kinkema M, Li X, Dong X (1999). Interaction of NPR1 with basic leucien zipper protein transcription factors that bind sequences required for salicylic acid induction of the PR-1 gene. Proceedings of the National Academy of Sciences of the United States of America 96:6523-6528.

Zhou JM, Trifa Y, Silva H, Pontier D, Lam E, Shah J, Klessig DF (2000). NPR1 differentially interacts with members of the TGA/OBF family of transcription factors that bind an element of the PR-1 gene required for induction by salicylic acid. Molecular Plant-Microbe Interactions 13:191-202.

https://www.arabidopsis.org/jsp/ChromosomeMap/tool.jpp

http://bar.utoronto.ca database

http://bioinformatics.psb.ugent.be/webtools/plantcare/html/bar.utoronta database

http://www.genemania.org

https://www.ncbi.nlm.nih.gov/Structure/cdd/wrpsb.cgi

http:/ /plantpan2 itps.ncku.edu.tw / promoter. php

http://www.phytozome.net/

http://string-db.org 